IMA Journal of Mathematical Control and Information Page 1 of 15 doi:10.1093/imamci/dnnxxx

\title{
Wirtinger-like Lyapunov-Krasovskii functionals for discrete-time delay systems
}

\author{
Alexandre Seuret and Emilia Fridman \\ LAAS - CNRS, Université de Toulouse, CNRS, Toulouse, France, \\ Tel Aviv University, Tel Aviv, Israel.
}

[Received on 18 February 2005]

\begin{abstract}
Time-dependent Lyapunov functionals appeared to be very efficient for sampled-data systems. In [14], new Lyapunov functionals were constructed for sampled-data control in the presence of a constant input delay. The construction of these functionals was based on Wirtinger's inequality leading to simplified and efficient stability conditions in terms of Linear Matrix Inequalities (LMIs). In the present paper we extend the latter results to the discrete-time sampled-data systems. We show that the proposed approach is less conservative on some examples with a lower number of decision variables.
\end{abstract}

Keywords: Discrete-time delay systems, sampled-data, Lyapunov-Krasovskii functional, Wirtinger inequality

\section{Introduction}

Sampled-data systems have been studied extensively over the past decades (see e.g. [1, 6, 17, 18, $8]$ and the references therein). Modeling of continuous-time systems with digital control in the form of continuous-time systems with time-varying delay ([16]) and the extension of Krasovskii method to systems with fast varying delays (without any constraints on the delay derivative as in [7]) and to discontinuous delays ([6]) have allowed the development of the time-delay approach to sampled-data and to network-based control (see Section 7 of [4] for details).

Till [3] the conventional time-independent Lyapunov functionals $V\left(x_{t}, \dot{x}_{t}\right)$ for systems with fastvarying delays were applied to sampled-data systems ([6]). These functionals did not take advantage of the sawtooth evolution of the delays induced by sampled-and-hold. The latter drawback was removed in [3] and [20], where time-dependent Lyapunov functionals (inspired by [18]) were constructed for sampled-data systems. A different time-dependent Lyapunov functional was suggested in [14] which is based on Wirtinger's inequality (see for instance [15] and [12]):

Let $z(t):(a, b) \rightarrow \mathbb{R}^{n}$ be absolutely continuous with $\dot{z} \in L_{2}[a, b]$ and $z(a)=0$. Then for any $n \times n$ matrix $W>0$ Wirtinger's inequality holds:

$$
\int_{a}^{b} \dot{z}^{T}(\xi) W \dot{z}(\xi) d \xi \geqslant \frac{\pi^{2}}{4(b-a)^{2}} \int_{a}^{b} z^{T}(\xi) W z(\xi) d \xi .
$$

The Wirtinger-based LMI is a single LMI with fewer decision variables than the LMIs of [3, 20]. More important, differently from the Lyapunov functionals of [3] and [20], the extension of the Wirtingerbased Lyapunov functionals to a more general sampled-data system in the presence of a constant input/output delay leads to efficient stability conditions (see e.g.[14]).

In the present paper, we aim at extending the results of [14] to discrete-time sampled-data systems. Unlike the continuous-time case, the discrete-time formulation has surprisingly attracted only few attention in the literature even if the formulation represents an efficient way to model the dynamics of 
discrete-time systems subject to control packet losses. The problem of packet losses indeed appears in many applications of networked control systems (see for instance [11] and [24]). As in the continuoustime case, the Wirtinger-based Lyapunov functionals essentially reduce the numerical complexity of the resulting LMIs leading in some examples to less restrictive conditions. Similarly to the continuous-time case, discrete-time sampled-data can be seen as a discrete-time system subject to a particular timevarying delay, for which there exist many stability conditions (see e.g. [10, 23, 13]). However, such approaches do not account accurately the particularities of the sawtooth delay.

In the continuous-time case, the analysis of this class of functionals is made possible by considering that the functionals do not grow at the sampling instants. A translation of such analysis in the discretetime framework is not easy and requires a dedicated analysis.

The paper is organized as follows. Section 2 describes the problem formulation. Section 3 shows some preliminary summation inequalities including a Wirtinger's and Jensen's inequality as well as a recent summation inequality that includes the Jensen's inequality as a consequence. This last inequality is the counter part of the Wirtinger-based inequality provided in [21]. Section 4 presents the main results on the stability analysis of discrete-time sampled-data systems. Section 6 shows the efficiency of the proposed method on some examples. Finally Section 7 draws some conclusions.

Notations: Throughout the paper, $\mathbb{Z}(\mathbb{N})$ denotes the set of (positive) integers, $\mathbb{R}^{n}$ the $n$-dimensional Euclidean space with vector norm $|\cdot|, \mathbb{R}^{n \times m}$ the set of all $n \times m$ real matrices. For any symmetric matrix $P \in \mathbb{R}^{n \times n}$, the notation $P>0$ (or $P<0$ ) means that $P$ is positive (or negative) definite. The set $\mathbb{S}_{n}^{+}$refers to the set of symmetric positive definite matrices. For any matrices $A, B$ in $\mathbb{R}^{n \times n}$, the notation $\operatorname{diag}(A, B)$ denotes the block diagonal matrix $\left[\begin{array}{ll}A & 0 \\ * & B\end{array}\right]$. For any square matrix, $\operatorname{He}(A)$ stands for $A+A^{T}$. Along the paper, for any real number $a<b$, the notation $[a, b]_{\mathbb{Z}}$ denotes $[a, b] \cap \mathbb{Z}$. The same notations will also hold for open intervals.

\section{Problem formulation}

Consider a linear discrete-time time-delay system of the form:

$$
\begin{cases}x(t+1)=A x(t)+A_{d} x\left(t_{k}-h\right), & \forall k \in\left[k_{i}, k_{i+1}\right)_{\mathbb{Z}} \\ x(\theta)=x_{0}(\theta), & \forall \theta \in[-h, 0] .\end{cases}
$$

where $x(t) \in \mathbb{R}^{n}$ is the state vector, $x_{0}$ is the initial condition and $A, A_{d} \in \mathbb{R}^{n \times n}$ are constant matrices. The delay $h \in \mathbb{N}$ is assumed to be constant and known and the sequence of integers $\left\{t_{k}\right\}_{k \in \mathbb{N}} \subset \mathbb{N}$ satisfies

$$
\begin{aligned}
\forall k \in \mathbb{N}, \quad & t_{k+1}-t_{k} \in\left[1, T_{\max }\right] \mathbb{Z}, \\
& \lim _{k \rightarrow+\infty} t_{k}=+\infty .
\end{aligned}
$$

Following the principles of the input delay approach for discrete-time systems, we can define an input delay function $\tau$ given by

$$
\begin{aligned}
& \tau(t)=t-t_{k}, \quad \forall t \in\left[t_{k}, t_{k+1}\right)_{\mathbb{Z}}, \\
& \tau(t+1)=\left\{\begin{array}{lll}
\tau(t)+1 & \text { if } & t<t_{k+1} \\
0 & \text { if } & t=t_{k+1}
\end{array}\right.
\end{aligned}
$$

The system (2.1) can then be rewritten as a discrete-time system subject to a time-varying delay. The delay function can be seen as the discrete-time version of the sawtooth delay function considered in [6] for continuous-time sampled-data systems.

$$
\left\{\begin{array}{l}
x(t+1)=A x(t)+A_{d} x(t-h-\tau(t)), \quad \forall t \in\left[t_{k}, t_{k+1}\right)_{\mathbb{Z}} \\
x(\theta)=x_{0}(\theta), \forall \theta \in[-h, 0]_{\mathbb{Z}}
\end{array}\right.
$$


where the delay $h$ is constant and where the sampling delay $\tau(t)$ is given in (2.3). In this paper, we aim at providing stability conditions for this peculiar class of systems using a method based on discontinuous Lyapunov functionals. The present article can be seen as the discrete-time counterpart of the recent article [14]. To this end, we will provide a stability analysis of such class of systems, where novel Wirtinger-based terms are added to "nominal" Lyapunov functionals for the stability analysis of the discrete-time systems with the constant delay $h$.

\section{Preliminaries on summation inequalities}

\subsection{Discrete-time Wirtinger inequality}

Wirtinger inequalities are integral inequalities issued from the Fourier analysis. The continuous-time versions of this inequality have already shown their potential for the stability analysis of partial differential equation ([5]), sampled-data systems ([14]) or time-delay systems ([21]). In the present article we aim at showing that this class of inequalities also serves for the stability analysis of discrete-time systems. Indeed a discrete-time version of these inequalities have been extended to the discrete-time framework. It is stated in the following lemma taken from [2].

LEMmA 3.1 For a given $N \in \mathbb{N}_{\geqslant 0}$, consider a sequence of $N$ real scalars $x_{0}, x_{1}, \ldots, x_{N}$ such that $x_{0}=0$. Then, the following inequality holds

$$
\sum_{i=0}^{N-1}\left(x_{i}-x_{i+1}\right)^{2} \geqslant \lambda_{N}^{2} \sum_{i=0}^{N-1} x_{i}^{2}
$$

where $\lambda_{N}=2 \sin (\pi /(2(2 N+1)))$.

A straightforward corollary of this lemma is provided for $n$-dimensional sequences $z$ and is stated below.

COROLlARY 3.1 For a given $N \in \mathbb{N}_{\geqslant 0}$, consider a sequence of $N$ real $n$-dimensional vectors $z_{0}, z_{2}, \ldots, z_{N}$ such that $z_{0}=0$. Then, the following inequality holds, for any symmetric positive definite matrix $W \in \mathbb{S}_{+}^{n}$.

$$
\sum_{i=0}^{N-1}\left(z_{i}-z_{i+1}\right)^{T} W\left(z_{i}-z_{i+1}\right) \geqslant \lambda_{N}^{2} \sum_{i=0}^{N-1} z_{i}^{T} W z_{i}
$$

where $\lambda_{N}=2 \sin (\pi /(2(2 N+1)))$.

Proof. Since $W>0$, there exists an orthogonal matrix $U=\left[\begin{array}{cccc}U_{1}^{T} & U_{2}^{T} & \ldots & U_{n}^{T}\end{array}\right]^{T}$ and a positive definite diagonal matrix $\Delta=\operatorname{diag}\left(\Delta_{1}, \Delta_{2}, \ldots \Delta_{n}\right)$ such that $W=U^{T} \Delta U$. It holds

$$
\begin{aligned}
\lambda_{N}^{2} \sum_{i=0}^{N-1} z_{i}^{T} W z_{i} & =\lambda_{N}^{2} \sum_{i=0}^{N-1} \sum_{j=1}^{n}\left(U_{j} z_{i}\right)^{T} \Delta_{j}\left(U_{j} z_{i}\right) \\
& =\sum_{j=1}^{n} \Delta_{j} \lambda_{N}^{2} \sum_{i=0}^{N-1}\left(U_{j} z_{i}\right)^{2}
\end{aligned}
$$

Following the same procedure, we also have

$$
\sum_{i=0}^{N-1}\left(z_{i}-z_{i+1}\right)^{T} W\left(z_{i}-z_{i+1}\right)=\sum_{j=1}^{n} \Delta_{j} \sum_{i=0}^{N-1}\left(U_{j}\left(z_{i}-z_{i+1}\right)\right)^{2}
$$


Since the vector $z_{0}=0$, the scalar $U_{j} z_{0}$ is zero for all $j=1, \ldots, n$. Hence the Wirtinger inequality in Lemma 3.1 ensures that

$$
\sum_{i=0}^{N-1}\left(U_{j}\left(z_{i}-z_{i+1}\right)\right)^{2} \geqslant \lambda_{N}^{2} \sum_{i=0}^{N-1}\left(U_{j} z_{i}\right)^{2}, \quad j=1, \ldots, n .
$$

Finally computing the sum over $j=1, \ldots, n$ of the previous inequality weighted by $\Delta_{j}$ leads to

$$
\sum_{j=1}^{n} \Delta_{j} \sum_{i=0}^{N-1}\left(U_{j}\left(z_{i}-z_{i+1}\right)\right)^{2} \geqslant \sum_{j=1}^{n} \Delta_{j} \lambda_{N}^{2} \sum_{i=0}^{N-1}\left(U_{j} z_{i}\right)^{2}
$$

which is equivalent to (3.2) thanks to (3.3) and (3.4).

\subsection{Summation inequalities}

In this section, two summation inequalities are recalled. The first one is the Jensen inequality and is stated here.

LEMMA 3.2 For a given symmetric positive definite matrix $Z \in \mathbb{S}_{+}^{n}$, any sequence of discrete-time variable $x$ in $[-h, 0]_{\mathbb{Z}} \rightarrow \mathbb{R}^{n}$, where $h \geqslant 1$, the following inequality holds:

$$
\sum_{i=-h+1}^{0} y^{T}(i) Z y(i) \geqslant \frac{1}{h} \Theta_{0}^{T} Z \Theta_{0}
$$

where $y(i)=x(i)-x(i-1)$ and $\Theta_{0}=x(0)-x(-h)$.

The second lemma is a recent inequality proposed in [22] that extends the Wirtinger-based integral inequality (see [21]) to the discrete-time case.

Lemma 3.3 For a given symmetric positive definite matrix $Z \in \mathbb{S}_{+}^{n}$, any sequence of discrete-time variable $x$ in $[-h, 0]_{\mathbb{Z}} \rightarrow \mathbb{R}^{n}$, where $h \geqslant 1$, the inequality

$$
\sum_{i=-h+1}^{0} y^{T}(i) Z y(i) \geqslant \frac{1}{h}\left[\begin{array}{l}
\Theta_{0} \\
\Theta_{1}
\end{array}\right]^{T}\left[\begin{array}{cc}
Z & 0 \\
0 & 3\left(\frac{h+1}{h-1}\right) Z
\end{array}\right]\left[\begin{array}{c}
\Theta_{0} \\
\Theta_{1}
\end{array}\right],
$$

holds where

$$
\begin{aligned}
y(i) & =x(i)-x(i-1), \\
\Theta_{0} & =x(0)-x(-h), \\
\Theta_{1} & =x(0)+x(-h)-\frac{2}{h+1} \sum_{i=-h}^{0} x(i) .
\end{aligned}
$$

Proof. The proof is provided in [22] and is therefore omitted.

REMARK 3.1 The inequality provided in Lemma 3.3 implies

$$
\sum_{i=-h+1}^{0} y^{T}(i) Z y(i) \geqslant \frac{1}{h} \Theta_{0}^{T} Z \Theta_{0},
$$

which is exactly the Jensen summation inequality. Therefore, Lemma 3.3 is less conservative than the celebrated Jensen inequality since a positive quantity is added in the right-hand side of the inequalities. 


\section{Stability analysis}

\subsection{Wirtinger-based functional}

In this section, we aim at proposing a new functional to deal with the discrete-time sampled-data system (2.4) by an appropriate use of the discrete time Wirtinger inequality resumed in Lemma 3.1. This contribution is proposed in the following lemma.

Lemma 4.1 Consider the following Lyapunov functional, for a given matrix $W \in \mathbb{S}_{+}^{n}$, a given $k \in \mathbb{N}$ and for all $t \in\left[t_{k}, t_{k+1}\right)_{\mathbb{Z}}$

$$
V_{W}\left(x_{t}\right)=\sum_{i=t_{k}-h}^{t-1} y^{T}(i) W y(i)-\lambda_{T}^{2} \sigma\left(t, x_{t}\right)
$$

where

$$
\begin{aligned}
& y(i) \quad=x(i+1)-x(i), \quad \forall i \in \mathscr{N} \\
& \lambda_{T}=2 \sin \left(\frac{\pi}{2\left(2 T_{\max }+1\right)}\right) \\
& \sigma\left(t, x_{t}\right)= \begin{cases}\sum_{i=t_{k}}^{t-1} v(i) W v(i), & t \in\left[t_{k}+1, t_{k+1}-1\right] \\
0, & t=t_{k},\end{cases} \\
& v(i) \quad=x(i-h)-x\left(t_{k}-h\right), \quad i \in\left[t_{k}, t_{k+1}-1\right] .
\end{aligned}
$$

Then, the forward difference of the functional $V_{W}$ satisfies the inequality

$$
\Delta V_{W}\left(x_{t}\right) \leqslant(x(t+1)-x(t))^{T} W(x(t+1)-x(t))-\lambda_{T}^{2} v^{T}(t) W v(t)
$$

holds, for all $t \in\left[t_{k}, t_{k+1}\right)_{\mathbb{Z}}$, and for any sampling satisfying (2.2).

Proof. For a given $k \in \mathbb{N}$, consider first $t \in\left[t_{k}, t_{k+1}-2\right]_{\mathbb{Z}}$. Then the computation of $\Delta V_{W}$ straightforwardly leads, for all $t \in\left[t_{k}, t_{k+1}-2\right]_{\mathbb{Z}}$, to

$$
\begin{aligned}
\Delta V_{W}\left(x_{t}\right) & =\sum_{i=t_{k}-h}^{t} y^{T}(i) W y(i)-\sum_{i=t_{k}-h}^{t-1} y^{T}(i) W y(i)-\lambda_{T}^{2}\left(\sigma\left(t+1, x_{t+1}\right)-\sigma\left(t, x_{t}\right)\right) \\
& =y^{T}(t) W y(t)-\lambda_{T}^{2}\left(\sigma\left(t+1, x_{t+1}\right)-\sigma\left(t, x_{t}\right)\right) .
\end{aligned}
$$

From the definition of $\sigma\left(t, x_{t}\right)$, it is easy to see that, if $t \neq t_{k}$, we have

$$
\sigma\left(t+1, x_{t+1}\right)-\sigma\left(t, x_{t}\right)=\sum_{i=t_{k}}^{t_{k}} v(i) W v(i)-0=v^{T}(t) W v(t),
$$

and if $t=t_{k}$

$$
\sigma\left(t_{k}+1, x_{t_{k}+1}\right)-\sigma\left(t_{k}, x_{t_{k}}\right)=\sum_{i=t_{k}}^{t} v(i) W v(i)-\sum_{i=t_{k}}^{t-1} v(i) W v(i)=v^{T}\left(t_{k}\right) W v\left(t_{k}\right)
$$

This ensures that, for all $t \in\left[t_{k}, t_{k+1}-2\right]_{\mathbb{Z}}$, the following equality holds

$$
\Delta V_{W}\left(x_{t}\right)=(x(t+1)-x(t))^{T} W(x(t+1)-x(t))-\lambda_{T}^{2} v^{T}(t) W v(t) .
$$


Consider now the remaining case $t=t_{k+1}-1$. The computation of $\Delta V_{W}$ leads to

$$
\begin{aligned}
\Delta V_{W}\left(x_{t}\right) & =\sum_{i=t_{k+1}-h}^{t_{k+1}-1} y^{T}(i) W y(i)-\sum_{i=t_{k}-h}^{t_{k+1}-2} y^{T}(i) W y(i)-\lambda_{T}^{2}\left(0-\sum_{i=t_{k}-h}^{t_{k+1}-h-2} v^{T}(i) W v(i)\right) \\
& =y^{T}(t) W y(t)-\lambda_{T}^{2} v^{T}(t) W v(t)-\psi
\end{aligned}
$$

where

$$
\psi=\sum_{i=t_{k}-h}^{t_{k+1}-h-1} y^{T}(i) W y(i)-\lambda_{T}^{2} \sum_{i=t_{k}-h}^{t_{k+1}-h-1} v^{T}(i) W v(i) .
$$

By noting that

$$
\begin{array}{ll}
v\left(t_{k}\right) & =0, \quad \forall k \in \mathbb{N} \\
v(i+1)-v(i) & =y(i), \quad \forall i \in\left[t_{k}, t_{k+1}\right)_{\mathbb{Z}}, \\
t_{k+1}-t_{k} & \leqslant T_{\max }, \quad \forall k \in \mathbb{N},
\end{array}
$$

the assumptions of the Wirtinger inequality in Corollary 3.1 are satisfied, which guarantees that $\psi \geqslant 0$. It thus holds that, for $t=t_{k+1}-1$

$$
\Delta V_{W}\left(x_{t}\right) \leqslant(x(t+1)-x(t))^{T} W(x(t+1)-x(t))-\lambda_{T}^{2} v^{T}(t) W v(t) .
$$

Then, combining (4.4) and (4.6) proves the result.

Note that inequality (4.6) is actually an equality when $t \neq t_{k+1}-1$ and is an inequality only when $t=t_{k+1}-1$. The computation of this inequality only relies on the computation of the forward increment of functional $V_{W}$ and the use of the Wirtinger inequality.

The objective in the remainder of the paper is to include this functional in the stability analysis of discrete sampled-data systems. Next, we will propose two stability theorems which rely on the use of the Jensen inequality and on Lemma 3.3.

\subsection{Jensen-based Theorem}

The following theorem holds

THEOREM 4.1 For given $h$ and $T_{\max }$ in $\mathbb{N}$, assume that there exist $n \times n$ matrices $P, Q, Z$ and $W \in \mathbb{S}_{+}^{n}$ such that the LMI condition

$$
\Pi\left(T_{\max }, h\right)<0
$$

holds where

$$
\begin{array}{rlrl}
\Pi\left(T_{\max }, h\right) & =\left[\begin{array}{ccc}
Q & 0 & 0 \\
* & -Q & 0 \\
* & * & -\lambda_{T}^{2} W
\end{array}\right]+M_{1}^{T} P M_{1}-M_{2}^{T} P M_{2}+M_{0}^{T}\left(W+h^{2} Z\right) M_{0}-M_{3}^{T} Z M_{3}, \\
M_{0} & =\left[\begin{array}{ccc}
A-I & A_{d} & -A_{d}
\end{array}\right], & M_{1}=\left[\begin{array}{ccc}
A & A_{d} & -A_{d}
\end{array}\right], \\
M_{2} & =\left[\begin{array}{ll}
I & 0
\end{array}\right], & M_{3}=\left[\begin{array}{lll}
I & -I & 0
\end{array}\right], \\
\lambda_{T} & =2 \sin \left(\frac{\pi}{2\left(2 T_{\max }+1\right)}\right) . &
\end{array}
$$

Then system (2.4) is asymptotically stable for the constant delay $h$ and any aperiodic sampling satisfying (2.2). 
Proof. Consider the functional

$$
V\left(x_{t}\right)=V_{1}\left(x_{t}\right)+V_{W}\left(x_{t}\right)
$$

where the functional $V_{W}$ have been defined previously. The fucntional $V_{1}$ is build to assess stability of the delayed term $A_{1} x(t-h)$ which appears in equation (2.4). Indeed a classical functional for discrete-time delay system is given by

$$
V_{1}\left(x_{t}\right)=x^{T}(t) P x(t)+\sum_{i=t-h}^{t-1} x^{T}(i) Q x(i)+h \sum_{i=-h+1}^{0} \sum_{j=t+i-1}^{t-1} y^{T}(j) Z y(j),
$$

where $y(i)=x(i+1)-x(i)$. Define the increment of the Lyapunov-Krasovskii functional as follows $\Delta V\left(x_{t}\right)=V\left(x_{t+1}\right)-V\left(x_{t}\right)$. From Lemma 4.1, we show that

$$
\begin{aligned}
\Delta V\left(x_{t}\right)= & x^{T}(t+1) P x(t+1)-x^{T}(t) P x(t)+x^{T}(t) Q x(t)-x^{T}(t-h) Q x(t-h) \\
& +h^{2}(x(t+1)-x(t))^{T} Z(x(t+1)-x(t))+(x(t+1)-x(t))^{T} W(x(t+1)-x(t)) \\
& -\lambda_{T}^{2} v^{T}(t) W v(t)-h \sum_{j=t-h}^{t-1} y^{T}(j) Z y(j) .
\end{aligned}
$$

Applying Jensen's inequality to the summation term ensures that

$$
\begin{aligned}
\Delta V\left(x_{t}\right) \leqslant & x^{T}(t+1) P x(t+1)-x^{T}(t) P x(t)+x^{T}(t) Q x(t)-x^{T}(t-h) Q x(t-h) \\
& +h^{2}(x(t+1)-x(t))^{T} Z(x(t+1)-x(t))-(x(t)-x(t-h))^{T} Z(x(t)-x(t-h)) \\
& +(x(t+1)-x(t))^{T} W(x(t+1)-x(t))-\lambda_{T}^{2} v^{T}(t) W v(t) .
\end{aligned}
$$

It follows from the previous calculations that

$$
\Delta V\left(x_{t}\right) \leqslant\left[\begin{array}{c}
x(t) \\
x(t-h) \\
v(t)
\end{array}\right]^{T} \Pi\left(T_{\max }, h\right)\left[\begin{array}{c}
x(t) \\
x(t-h) \\
v(t)
\end{array}\right]
$$

Then asymptotic stability results from the condition $\Pi(T, h)<0$, which concludes the proof.

\subsection{Improved stability Theorem}

As it was noticed in [22], the conservatism induces by the Jensen inequality can be notably reduced by considering the refined summation inequality provided in Lemma 3.3. The resulting analysis leads to the following theorem.

THEOREM 4.2 For given $h$ and $T_{\max }$ in $\mathbb{N}$, assume that there exist a $2 n \times 2 n$ matrix $P>0$ and $n \times n$ matrices $Q>0, Z>0$ and $W>0$ such that the LMI condition

$$
\Phi\left(T_{\max }, h\right)<0
$$


holds where

$$
\begin{aligned}
& \Phi\left(T_{\max }, h\right)=\left[\begin{array}{cccc}
Q & 0 & 0 & 0 \\
0 & -Q & 0 & 0 \\
0 & 0 & 0 & 0 \\
0 & 0 & 0 & -\lambda_{T}^{2} W
\end{array}\right]+N_{1}^{T} P N_{1}-N_{2}^{T} P N_{2}+N_{0}^{T}\left(W+h^{2} Z\right) N_{0}-N_{3}^{T} \tilde{Z} N_{3} \\
& N_{0}=\left[\begin{array}{llll}
A-I & A_{d} & 0 & -A_{d}
\end{array}\right] \\
& N_{1}=\left[\begin{array}{cccc}
A & A_{d} & 0 & -A_{d} \\
0 & -I & (h+1) I & 0
\end{array}\right] \\
& N_{2}=\left[\begin{array}{cccc}
I & 0 & 0 & 0 \\
-I & 0 & (h+1) I & 0
\end{array}\right] \\
& N_{3}=\left[\begin{array}{cccc}
I & -I & 0 & 0 \\
I & I & -2 I & 0
\end{array}\right] \\
& \tilde{Z}=\left[\begin{array}{cc}
Z & 0 \\
0 & 3 \frac{h+1}{h-1} Z
\end{array}\right] \\
& \lambda_{T}=2 \sin \left(\frac{\pi}{2\left(2 T_{\max }+1\right)}\right) \text {. }
\end{aligned}
$$

Then system (2.4) is asymptotically stable for the constant delay $h$ and any aperiodic sampling satisfying (2.2).

Proof. Consider the functional

$$
V\left(x_{t}\right)=V_{2}\left(x_{t}\right)+V_{W}\left(x_{t}\right)
$$

where we use the same definition for the functional $V_{W}$ as in Theorem 4.1. In order to fully take advantages of the summation inequality provided in Lemma 3.3, we select the following functional $V_{2}$ given by

$$
\begin{aligned}
V_{2}\left(x_{t}\right)= & {\left[\sum_{i=t-h}^{x-1} x(t)\right]^{T} P\left[\sum_{i=t-h}^{t-1} x(i)\right]+\sum_{i=t-h}^{t-1} x^{T}(i) Q x(i) } \\
& +h \sum_{i=-h+1}^{0} \sum_{j=t+i-1}^{t-1} y^{T}(j) Z y(j),
\end{aligned}
$$

where $y(i)=x(i+1)-x(i)$. This functional has been build according to the method provided in [22]. The forward difference of the Lyapunov-Krasovskii functional yields

$$
\begin{aligned}
& \Delta V\left(x_{t}\right)=\left[\begin{array}{c}
x(t+1) \\
\sum_{i=t-h+1}^{t} x(i)
\end{array}\right]^{T} P\left[\begin{array}{c}
x(t+1) \\
\sum_{i=t-h+1}^{t} x(i)
\end{array}\right]-\left[\sum_{i=t-h}^{t-1} x(i)\right]^{T} P\left[\begin{array}{c}
x(t) \\
t-1 \\
\sum_{i=t-h} x(i)
\end{array}\right] \\
& +x^{T}(t) Q x(t)-x^{T}(t-h) Q x(t-h)+h^{2}(x(t+1)-x(t))^{T} Z(x(t+1)-x(t)) \\
& -h \sum_{j=t-h}^{t-1} y^{T}(j) Z y(j)
\end{aligned}
$$

Define the $\xi(t)=\frac{1}{h+1} \sum_{i=t-h}^{t} x(i)$ and applying the summation provided in Lemma 3.3 to the last term 
ensures that

$$
\begin{aligned}
\Delta V\left(x_{t}\right) \leqslant & {\left[\begin{array}{c}
x(t+1) \\
(h+1) \xi(t)-x(t-h)
\end{array}\right]^{T} P\left[\begin{array}{c}
x(t+1) \\
(h+1) \xi(t)-x(t)
\end{array}\right] } \\
& -\left[\begin{array}{c}
x(t) \\
(h+1) \xi(t)-x(t)
\end{array}\right]^{T} P\left[\begin{array}{c}
x(t) \\
(h+1) \xi(t)-x(t)
\end{array}\right] \\
& +h^{2}(x(t+1)-x(t))^{T} Z(x(t+1)-x(t))-(x(t)-x(t-h))^{T} Z(x(t)-x(t-h)) \\
& -3\left(\frac{h+1}{h-1}\right)(x(t)+x(t-h)-2 \xi(t))^{T} Z(x(t)+x(t-h)-2 \xi(t)) .
\end{aligned}
$$

It follows from the previous calculations that

$$
\Delta V\left(x_{t}\right) \leqslant\left[\begin{array}{c}
x(t) \\
x(t-h) \\
\xi(t) \\
v(t)
\end{array}\right]^{T} \Phi\left(T_{\max }, h\right)\left[\begin{array}{c}
x(t) \\
x(t-h) \\
\xi(t) \\
v(t)
\end{array}\right] .
$$

Then asymptotic stability results from the condition $\Phi\left(T_{\max }, h\right)<0$, which concludes the proof.

REMARK 4.1 In the previous developments, we only focussed on the case of discrete-time delay systems with a single delay and a single sampling. However, the methodology can be extended to the case of multiple delays and multiple sampling by introducing additional functional terms. For the sake of consistency, this problem is not addressed in this paper.

\subsection{Comparison with approaches from the literature}

In the present paper, we consider functionals of the form

$$
V\left(x_{t}\right)=V_{1}\left(x_{t}\right)+V_{W}\left(x_{t}\right)
$$

where the functional $V_{1}$ (or $V_{2}$ ) aims at assessing the stability of system (2.1) without sampling and where the functional $V_{W}\left(x_{t}\right)$ aims at ensuring the robustness with respect to the sampling. In [22], the functional can also be split into two parts where the first one is again the same $V_{1}$ (or $V_{2}$ ) but the second part is related to the time-varying delay case. Therefore the conditions provided in [22] only address the stability of the system driven by

$$
x(t+1)=A x(t)+A_{d} x(t-h(k)),
$$

where the delay $h(k)$ can take any values between $h$ and $h+T$, without respecting the constraint imposed in this paper on the sawtooth form of the delay. Therefore the conditions provided in the present paper and the one provided in [22] does not treat the same problem. However, it is correct to say that the conditions of [22] guarantee stability of the sampled-data system (2.1) but also to a larger class of delay systems.

The idea of this paper is to propose a dedicated construction of the functional to cope with the stability analysis of sampled and delayed closed-loop system driven by (2.1).

\subsection{Example 1}

Consider the continuous time sampled-data system linear driven, for all $\mathbf{t} \in\left[k T_{0},(k+1) T_{0}\right)_{\mathbb{Z}}$ by

$$
\dot{x}(\mathbf{t})=A_{c} x(\mathbf{t})+B_{c d} K x\left((k-h) T_{0}\right),
$$




\begin{tabular}{|c|c|}
\hline Theorems & Complexity \\
\hline$[13]$ & $\left(3.5 n^{2}+2.5 n\right) \times 4 n$ \\
\hline$[22]$ & $\left(10 n^{2}+3.5 n\right) \times 12 n$ \\
\hline Theorem 4.1 & $\left(2 n^{2}+2 n\right) \times 3 n$ \\
\hline Theorem 4.2 & $\left(3.5 n^{2}+2.5 n\right) \times 4 n$ \\
\hline
\end{tabular}

Table 1. Complexity of the LMI conditions tested in the example

\begin{tabular}{|c|c|c|c|c|c|c|c|c|c|c|c|}
\hline$T_{\max }$ & 1 & 2 & 3 & 4 & 5 & 6 & 7 & 8 & 9 & 10 & 11 \\
\hline$[13]$ & 9 & 8 & 7 & 6 & 5 & 4 & 3 & 2 & 1 & - & - \\
\hline$[22]$ & 9 & 8 & 7 & 6 & 5 & 4 & 3 & 2 & 1 & - & - \\
\hline Theorem 4.1 & 9 & 8 & 7 & 6 & 5 & 4 & 4 & 3 & 2 & 1 & 1 \\
\hline Theorem 4.2 & 9 & 8 & 7 & 6 & 6 & 5 & 4 & 3 & 2 & 1 & 1 \\
\hline
\end{tabular}

Table 2. Results derived from various theorems showing the maximal admissible constant delay $h$ for several values of the sampling period for system with $T_{0}=0.1$

where $\mathbf{t}$ represents the continuous time and where

$$
A_{c}=\left[\begin{array}{cc}
0 & 1 \\
0 & -0.1
\end{array}\right], B_{c d}=\left[\begin{array}{cc}
0 & 0 \\
-0.375 & -1.15
\end{array}\right], \quad K=\left[\begin{array}{ll}
-0.375 & -1.15
\end{array}\right]
$$

and $T_{0}$ is the discretization period, $h$ is the delay and $k$ is a positive integer. The associated discretized system is given by discrete-time systems with delay given in (2.4) with the matrices

$$
A=e^{A_{c} T_{0}}, \quad A_{d}=\int_{0}^{T_{0}} e^{A_{c}\left(T_{0}-s\right)} d s B_{c d} K .
$$

The stability conditions provided in this paper and from the literature are tested on this system for several values of the discretization period $T_{0}=0.1$ and $T_{0}=0.01$. The results and a comparison with existing results from the literature are presented in Tables 2 and 3.

The stability conditions from $[13,22]$ address the problem of stability analysis of discrete-time systems subject to an unknown time-varying delay but which belongs to the interval $[h, h+T]$. To the best of our knowledge, these results are the most efficient conditions for the stability analysis of discrete systems with interval time-varying delays The sawtooth delay addressed in this paper is only a particular case of this more general class of time-varying delays.

Tables 2 and 3 show that our theorems essentially reduce the complexity of the conditions provided in $[13,22]$ leading to less conservative results.

Finally Figure 1 depicts the solutions of system (4.13) taken with a sampling period of the continuous time systems $T_{0}=0.1$ and the input delay $h=6$. From Table 2, the maximal length between two successive control update $t_{k+1}-t_{k}$ is upper bounded by 6 . Figure 1 shows two simulations of a periodic and an aperiodic implementation of the control input $u=K x\left(t_{k}-h\right)$ where it can be seen that the solutions of the systems remain stable in both cases. It is also worth noting that the system remains stable with the periodic implementation up to $T=12$, which means that the stability conditions resulting from the functional term related to the Wirtinger inequality are still conservative even if they already improve the condition issued from the time-varying delay case, which, again, allows assessing stability of the system with a larger class of delay functions than sawtooth delays. 


\begin{tabular}{|c|c|c|c|c|c|c|c|c|}
\hline$T_{\max }$ & 5 & 10 & 25 & 50 & 75 & 100 & 125 & 133 \\
\hline$[13]$ & 101 & 96 & 81 & 56 & 31 & 4 & - & - \\
\hline$[22]$ & 109 & 103 & 86 & 58 & 33 & 7 & - & - \\
\hline Theorem 4.1 & 102 & 98 & 85 & 65 & 45 & 25 & 7 & 1 \\
\hline Theorem 4.2 & 110 & 106 & 91 & 69 & 47 & 26 & 7 & 1 \\
\hline
\end{tabular}

Table 3. Results derived from various theorems showing the maximal admissible constant delay $h$ for several values of the sampling period for system with $T_{0}=0.01$
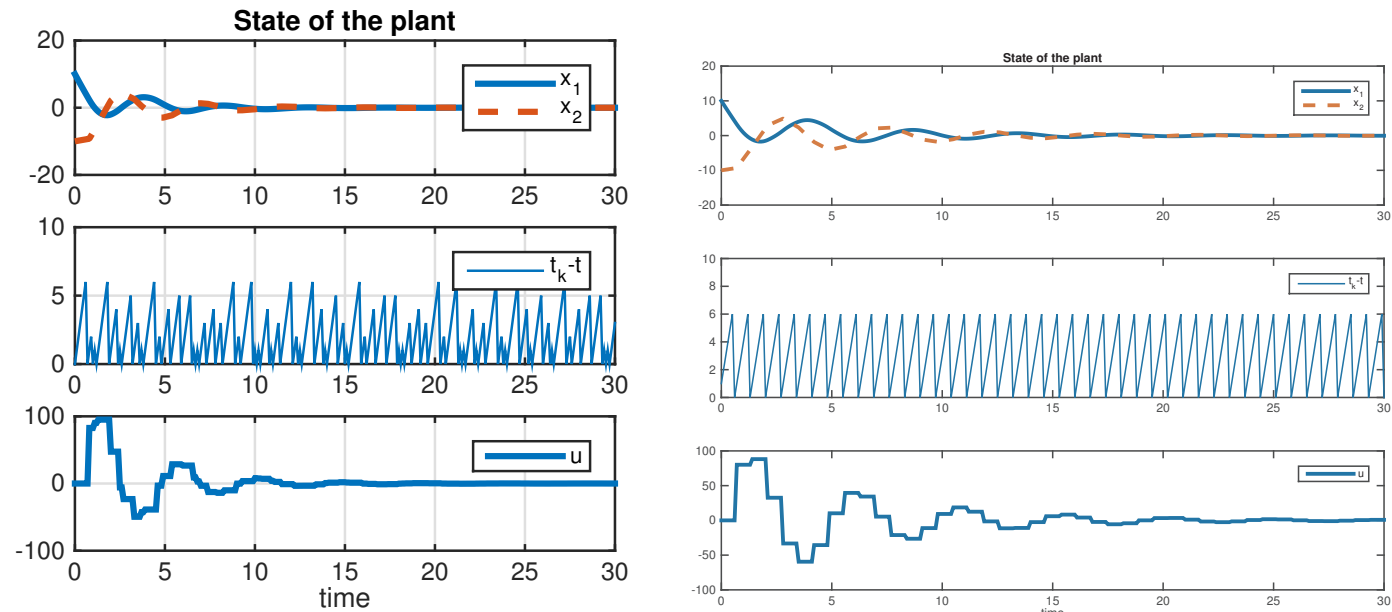

(a) Aperiodic sampling $T_{\max }=6$.

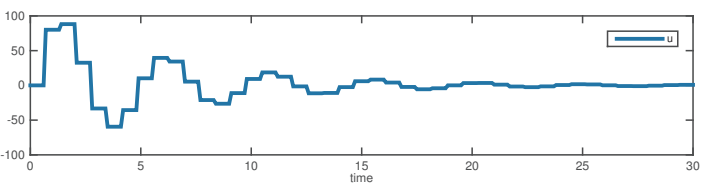

(b) Periodic sampling $T=6$.

FIG. 1. Example 1. Simulation results representing the state $x$ (top), the timer $\tau=t-t_{k}$ (middle), and the control inputs $u=$ $K x\left(t_{k}-h\right)$ (bottom), of System (4.13) with $T_{0}=0.1$ with $h=5, T_{\max }=6$ and aperiodic (a) and periodic (b) samplings.

\section{Model reduction and Predictor control}

\subsection{Definitions}

Consider the linear discrete-time system driven by

$$
\left\{\begin{array}{l}
x(t+1)=A x(t)+B u\left(t_{k}-h\right), \quad \forall t \in\left[t_{k}, t_{k+1}\right) \cap \mathbb{N} \\
x(\theta)=x_{0}(\theta), \quad \forall \theta \in[-h, 0] .
\end{array}\right.
$$

where $x(t) \in \mathbb{R}^{n}$ is the state vector, $x_{0}$ is the initial condition and $A, A_{d} \in \mathbb{R}^{n \times n}$ are constant matrices. with the initial condition $x_{0}$. The prediction-based approach consists in considering the following control law

$$
\begin{aligned}
& u(t)=K \bar{x}(t+h) \\
& \bar{x}(t+h)=A^{h} x(t)+\sum_{i=0}^{h-1} A^{h-i-1} B u(t-h+i)
\end{aligned}
$$

In this formulation, the vector $\bar{x}$ is the $h$-step ahead state prediction. 
Lemma 5.1 The closed-looped system (5.1) with the control scheme (5.2) can be expressed as

$$
z(t+1)=(A+B K) z(t)-A^{h} B K\left[z(t-h)-z\left(t_{k}-h\right)\right] .
$$

Proof. Define the new state $z(t)=\bar{x}(t+h)$ where $\bar{x}$ is given in (5.2). It holds

$$
\begin{aligned}
z(t+1) & =A^{h}\left(A x(t)+B u\left(t_{k}-h\right)\right)+\sum_{i=0}^{h-1} A^{h-i-1} B u(t+1-h+i) \\
& =A^{h}\left(A x(t)+B u\left(t_{k}-h\right)\right)+A \sum_{i=0}^{h-1} A^{h-i-1} B u(t-h+i)+B u(t)-A^{h} B u(t-h) \\
& \left.=A\left[A^{h} x(t)+\sum_{i=0}^{h-1} A^{h-i-1} B u(t-h+i)\right]+B u(t)+A^{h} B u\left(t_{k}-h\right)\right)-A^{h} B u(t-h) \\
& \left.=A z(t)+B u(t)+A^{h} B\left[u\left(t_{k}-h\right)\right)-u(t-h)\right]
\end{aligned}
$$

Finally, reinjecting the definition of $u=K z$ in the previous equation leads to the result.

\subsection{Stability conditions}

The following theorem holds

THEOREM 5.1 For a given controller gain $K$ and a given delay $h$, assume that there exists two $n \times n$ matrices $P>0$ and $W>0$ such that the LMI condition

$$
\left[\begin{array}{cccc}
-P & 0 & (A+B K-I)^{T} W & (A+B K)^{T} P \\
* & -\lambda_{T}^{2} W & -\left(A^{h} B K\right)^{T} W & -\left(A^{h} B K\right)^{T} P \\
* & * & -W & 0 \\
* & * & * & -P
\end{array}\right]<0
$$

holds where $\lambda_{T}=2 \sin \left(\frac{\pi}{2(2 T+1)}\right)$.

Proof. Consider the functional

$$
V_{t}\left(x_{t}\right)=x^{T}(t) P x(t)+V_{W}\left(x_{t}\right)
$$

where the functionals $V$ and $V_{W}$ have been defined previously. It follows from the previous calculations that

$$
\Delta V_{t}\left(x_{t}\right) \leqslant\left[\begin{array}{c}
x(t) \\
v(t)
\end{array}\right]^{T} \Psi_{2}\left[\begin{array}{l}
x(t) \\
v(t)
\end{array}\right]
$$

where

$$
\begin{aligned}
\Psi_{2}= & {\left[\begin{array}{cc}
-P & 0 \\
* & -\lambda_{T}^{2} W
\end{array}\right]+\left[\begin{array}{c}
(A+B K)^{T}-I \\
-\left(A^{h} B K\right)^{T}
\end{array}\right] W\left[\begin{array}{c}
(A+B K)^{T}-I \\
-\left(A^{h} B K\right)^{T}
\end{array}\right]^{T} } \\
& +\left[\begin{array}{c}
(A+B K)^{T} \\
-\left(A^{h} B K\right)^{T}
\end{array}\right] P\left[\begin{array}{c}
(A+B K)^{T} \\
-\left(A^{h} B K\right)^{T}
\end{array}\right]^{T}
\end{aligned}
$$

Then asymptotic stability results from the condition $\Psi_{2}<0$, which is equivalent to (5.4) by application of the Schur complement. 


\begin{tabular}{|c|c|c|c|c|c|c|c|c|c|c|c|}
\hline T & 1 & 2 & 3 & 4 & 5 & 6 & 7 & 8 & 9 & 10 & Number of decision Variables \\
\hline Theorem 5.1 & 3 & 7 & 10 & 13 & 15 & 16 & 17 & 19 & 20 & 21 & $3.5 n^{2}+2.5 n$ \\
\hline
\end{tabular}

Table 4. Evolution of the maximal admissible sampling period $T$ for several values of the input delay $h$

\subsection{Example 2}

Consider the linear discrete-time systems with delay given in (2.4) with the matrices taken from [9]

$$
A=\left[\begin{array}{cc}
0.8 & 0 \\
0.05 & 0.9
\end{array}\right], \quad A_{d}=\left[\begin{array}{cc}
-0.1 & 0 \\
-0.2 & -0.1
\end{array}\right]
$$

The results are presented in Table 4. One can see from this example that the robustness of the predictor control is reenforced for large delays. This means that the more the delay, the more the maximal allowable sampling period is obtained. A possible interpretation for such behavior is that the matrix $A_{0}$ is Schur stable. Therefore the matrix $A_{0}^{h}$ in the LMI conditions becomes smaller when the delay increases so that the contribution of $\lambda_{T}$ becomes sufficiently great to ensure robustness with respect to the sampling period.

A counter part of this numerical results, is that the performances of the closed loop systems may be affected. This means that increasing the delay $T$ for large values of $h$ would lead to power performances. In order to measure the performance degradation, one may look at $\mathscr{L}_{2}$ performance criteria or exponential stability criteria with guaranteed decay rate. For the latter solution, one would need to lightly modify the Wirtinger-based functional to account for exponential stability. This can be achieved following the idea developed for the continuous-time case in Lemma 1 of [19].

\section{Conclusions}

This paper addresses the stability analysis of discrete time sampled-data systems. The approach developed in this paper can be interpreted as the counterpart of the recent result on continuous-time systems from [14]. Two stability theorems have been provided and are tested on a simple example showing the efficiency of the method.

\section{References}

[1] T. Chen and B.A. Francis. Optimal Sampled-Data Control Systems. Springer-Verlag, London, 1995.

[2] Ky Fan, Olga Taussky, and John Todd. Discrete analogs of inequalities of wirtinger. Monatshefte für Mathematik, 59(2):73-90, 1955.

[3] E. Fridman. A refined input delay approach to sampled-data control. Automatica, 46:421-427, 2010.

[4] E. Fridman. Introduction to time-delay systems: analysis and control. Birkhauser, Systems and Control: Foundations and Applications, 2014.

[5] E. Fridman and Y. Orlov. Exponential stability of linear distributed parameter systems with timevarying delays. Automatica, 45(2):194-201, 2009. 
[6] E. Fridman, A. Seuret, and J. P. Richard. Robust sampled-data stabilization of linear systems: an input delay approach. Automatica, 40(8):1441-1446, 2004.

[7] E. Fridman and U. Shaked. Delay dependent stability and $H_{\infty}$ control: constant and time-varying delays. International Journal of Control, 76(1):48-60, 2003.

[8] H. Fujioka. Stability analysis of systems with aperiodic sample-and-hold devices. Automatica, 45:771-775, 2009.

[9] H. Gao, J. Lam, C. Wang, and Y. Wang. Delay-dependent output-feedback stabilisation of discretetime systems with time-varying state delay. IEE Proceedings-Control Theory and Applications, 151(6):691-698, 2004.

[10] Huijun Gao and Tongwen Chen. New results on stability of discrete-time systems with timevarying state delay. Automatic Control, IEEE Transactions on, 52(2):328-334, 2007.

[11] J. Hespanha, P. Naghshtabrizi, and Y. Xu. A survey of recent results in networked control systems. Proceedings of the IEEE, 95(1):138-162, 2007.

[12] W.D. Kammler. A first Course in Fourier Analysis. Cambridge University Press, Cambridge, 2007.

[13] J Liu and J Zhang. Note on stability of discrete-time time-varying delay systems. Control Theory \& Applications, IET, 6(2):335-339, 2012.

[14] K. Liu and E. Fridman. Wirtinger's inequality and Lyapunov-based sampled-data stabilization. Automatica, 48:102-108, 2012.

[15] K. Liu, V. Suplin, and E. Fridman. Stability of linear systems with general sawtooth delay. Special issue on Time-delay Systems in IMA Journal of Mathematical Control and Information, 27(4):419436, 2010.

[16] Yu. Mikheev, V. Sobolev, and E. Fridman. Asymptotic analysis of digital control systems,. Automation and Remote Control, 49:1175-1180, 1988.

[17] L. Mirkin. Some remarks on the use of time-varying delay to model sample-and-hold circuits. IEEE Transactions on Automatic Control, 52(6):1109-1112, 2007.

[18] P. Naghshtabrizi, J. Hespanha, and A. Teel. Exponential stability of impulsive systems with application to uncertain sampled-data systems. Systems and Control Letters, 57(5):378-385, 2008.

[19] A. Selivanov and E. Fridman. Observer-based input-to-state stabilization of networked control systems with large uncertain delays. Automatica, 74:63-70, 2016.

[20] A. Seuret. A novel stability analysis of linear systems under asynchronous samplings. Automatica, 48(1):177-182, 2012.

[21] A. Seuret and F. Gouaisbaut. Wirtinger-based integral inequality: Application to time-delay systems. Automatica, 49(9):2860-2866, 2013.

[22] A. Seuret, F. Gouaisbaut, and E. Fridman. Stability of discrete-time systems with time-varying delays via a novel summation inequality. To appear in IEEE Trans. on Automatic Control, 2015. 
[23] Hanyong Shao and Qing-Long Han. New stability criteria for linear discrete-time systems with interval-like time-varying delays. IEEE Trans. on Automatic Control, 56(3):619-625, 2011.

[24] S. Zampieri. Trends in networked control systems. In Proceedings of the 17th IFAC World Congress, Seoul, Korea, July 2008. 